\title{
Evaluation of renal transplantation patients followed-up at home by home care services during the COVID-19 pandemic in Turkey
}

Murat OZTURK ${ }^{1}$, Atilla SATIR ${ }^{1}$, Salih METIN²

${ }^{1}$ Department of Urology, University of Health Sciences Turkey Bursa Yuksek Ihtisas Training and Research Hospital, Bursa, TURKEY

2 Bursa Provincial Health Directorate, Bursa, TURKEY

\section{ABSTRACT}

Aim: The aim of this study was to evaluate the COVID-19 infection status of our renal transplant patients and who received immunosuppressive treatment during the pandemic period who were followed-up with home care services.

Methods: During the routine visits to our patients' homes during the pandemic, they were asked about their chronic disease histories, smoking and alcohol use and were asked triage questions about COVID19. PCR tests were applied to all transplant patients by collecting nasopharyngeal swabs.

Results: No COVID-19 positivity was detected in the PCR test for any of the patients who underwent renal transplantation in our clinic.

Conclusions: In this study, renal transplant patients were followed-up at home by home care services and thus kept away from the hospital environment. We assume that COVID-19 negativity during the first wave in our patients was a success of our home care services.

Keywords: COVID-19, home care service, PCR, transplantation, triage symptoms

\section{Corresponding Author:Murat OZTURK muratozturkdr@hotmail.com}

Received: October 15, 2021; Accepted: November 12, 2021; Published Online: December 31, 2021

Cite this article as: Ozturk, M., Satir, A., \& Metin, S. (2021). Determination of Social Support Status and Risk of Mental Illness in Patients Using Multiple Drugs in Primary Care. European Journal of Human Health 1(3), 98-104. 


\section{Introduction}

The number of COVID-19 cases exceeded 25 million and number of deaths exceeded 850 thousand worldwide as of the end of August 2020, during the eight-month period since the outbreak of the COVID19 pandemic (1). In Turkey, as of the end of August 2020, the number of cases exceeded 273 thousand and number of deaths exceeded 6400, since the first case seen on $11^{\text {th }}$ March 2020 (2).

Pregnant women, newborns, elderly people and patients with comorbidities such as diabetes mellitus, hypertension and cardiovascular diseases are more susceptible to COVID-19 infection and they are likely to present a more severe clinical picture that frequently requires intensive care. COVID-19 infection poses a special threat to dialysis patients (3). COVID19 infection presents specific difficulties for dialysis patients, and especially those who undergo hemodialysis (HD) in a clinic, not at home. Patients with uremia are specifically defenseless to infection and they can present more changes in clinical symptoms and infectivity. Intra-center HD increases the risk of spread of the infection significantly among the health care and facility personnel, patients, family members and others (4). As dialysis patients are in this high risk group, dialysis centers and clinics and the associated professionals must be ready to manage these patients safely, and to protect uninfected patients and personnel from this infection.

Renal transplant patients especially seem to be at high risk of Covid-19 disease due to chronic immunosuppression and comorbid circumstances. SARS-CoV2 infection is pushing the health care systems all around the world. It has been asserted that mortality of the disease is accompanied by comorbidities such as age, cardiovascular disease, diabetes, chronic respiratory disease, hypertension and cancer and these are associated with a worse prognosis. Immunosuppression and chronic renal disease may represent additional risk factors, but there are not yet any specific data. Since renal transplant patients severely experience the effects of immunosuppression, they must be accepted to be of high risk regardless of age. Considering that the infection may progress rapidly in these patients, their hospitalization indications should be evaluated accordingly. Moreover, considering that renal transplant patients may present atypical presentations (diarrhea, slight myalgia) besides classic symptoms, and false negative rates of the tests may be high, pulmonary CT scan indications are widely held, in case of doubt (5).

Transplant patients are at higher risk in terms of COVID-19 infection due to immunosuppression, underlying chronic renal failure and other comorbidities, especially diabetes and hypertension, which are accepted as important factors affecting the results (6). Depending on previous experience, the general opinion on the viral susceptibility of transplant recipients is that immunocompromised individuals, especially those with concomitant comorbidity, are under more serious risk of infection as their immune system is impaired. Although millions of global COVID19 cases have been reported worldwide, there is still a lack of information about its effects on renal transplant patients. The aim of this study was to evaluate the infection status of our renal transplant patients who received immunosuppressive treatment during the pandemic period and who suffered from comorbid diseases such as hypertension and diabetes and who were followed-up with home care services.

\section{Methods}

In our transplantation center there were 51 patients, 48 of whom were transplanted from cadavers and 
three from living donors. With the first COVID-19 case in Turkey as of March 2020, we immediately contacted our renal transplant patients and warned them not to come to the hospital unless it was necessary, and told them that their follow-up and treatment were going to be done by our home care services. They were asked to immediately call our center in case of the presence of even one of the COVID-19 symptoms. Home care services of these patients were performed routinely during this period. Patients who had complaints were taken to our hospital by home care cars and their necessary laboratory and scanning examinations were performed. PCR tests were applied to all our transplant patients by collecting nasopharyngeal swabs.

This study was conducted with 50 renal transplant patients who received transplantation at the Health Sciences University Bursa Yuksek Ihtisas Training and Research Hospital between July 2017 and February 2020 (19.34 \pm 9.25 months) due to chronic renal failure. Only one patient was excluded from the study because he was followed-up in another city. Ethics committee approval for the study was obtained from the local ethics committee. The study was performed in conformity with the Helsinki declaration. A consent form was received from all patients. 24 of the patients were male and 26 of them were female. The mean age of the patients was $44.12 \pm 11.64$ years.

All of our renal transplant recipients underwent immunosuppressive treatment at different doses depending on their renal functions. This treatment poses a risk for COVID-19 infection as well as for all infections. During the routine visits to our patients' homes during the pandemic, they were asked about their chronic disease histories, smoking and alcohol use and asked triage questions about COVID-19. The most frequent symptoms of COVID-19 among our renal transplant patients were: fever, coughing, shortness of breath, sore throat, myalgia and headache, and less frequent symptoms were: loss of taste or smell and diarrhea-nausea. The patients were examined in detail, laboratory and radiologic examinations were performed, and also COVID-PCR examinations were done by taking nasopharyngeal swabs.

\section{Statistical Analysis}

SPSS (IBM Corp. Released 2017. IBM SPSS Statistics for Windows, Version 25.0. Armonk, NY: IBM Corp.) Program was used for statistical analysis. Statistics for categorical variables are given as $\mathrm{n}(\%)$ values. Continuous variables are expressed with mean \pm standard deviation values.

\section{Results}

Fifty renal transplant patients participated in the study. Mean age of the participants was $44.12 \pm 11.64$. Gender distribution of the participants was 24 male and 26 female (Table 1). As a result of the study, no COVID-19 positivity was detected in the PCR test for any of the patients who underwent renal transplantation in our clinic.

It was observed that the most frequent chronic diseases of the participants were hypertension and diabetes mellitus (Table 1). Only in one case was coronary artery disease detected. Smoking was present in only three cases. There was no patient using alcohol. 
Table1: Demographic characteristics of the participants

\begin{tabular}{|l|l|l|}
\hline & $\mathrm{n}$ & $\%$ \\
\hline Male & 24 & 48 \\
\hline Female & 26 & 52 \\
\hline Coronary artery disease & 1 & 2 \\
\hline Hypertension & 34 & 68 \\
\hline Diabetes Mellitus & 7 & 14 \\
\hline Smokers & 7 & 6 \\
\hline Alcohol usage & 3 & 0 \\
\hline
\end{tabular}

While there were no symptoms in 25 of our patients, the most frequent symptoms were coughing $(n=12)$ and sore throat $(n=12)$ (Figure 1$)$. These symptoms were followed by myalgia in eight patients and headache and diarrhea in three patients each. Two of our patients presented three symptoms and nine of them presented two symptoms at the same time.

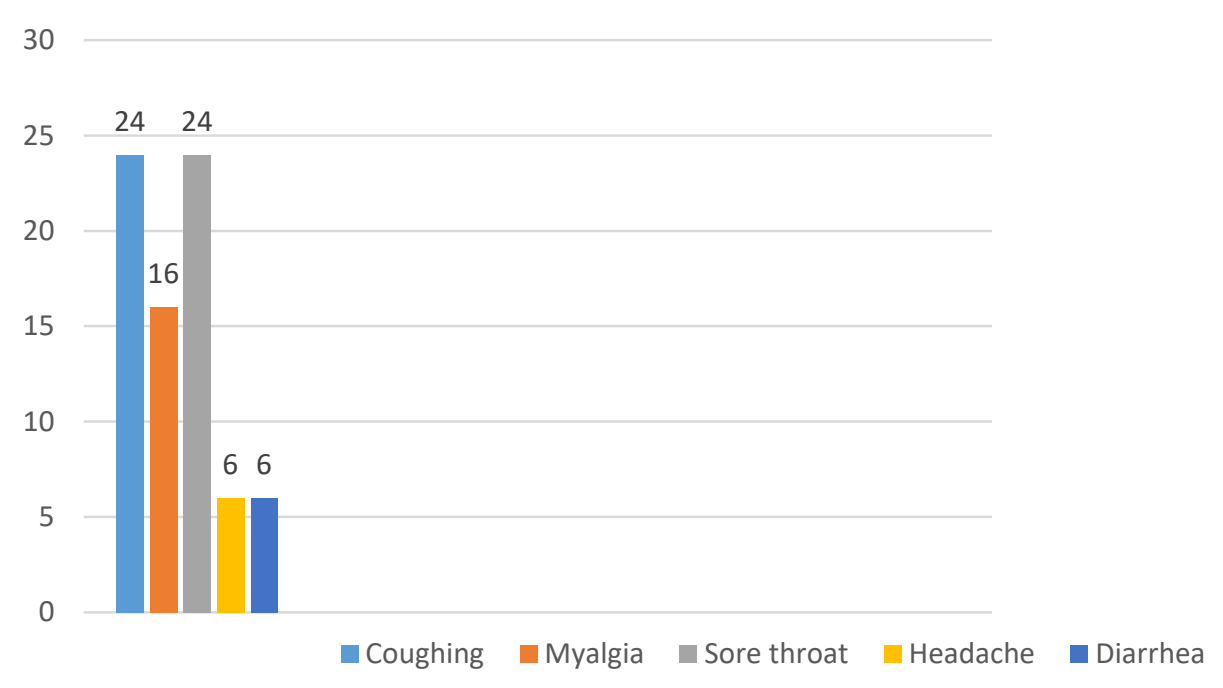

Figure1. Percentage distribution of Covid-19 symptoms in renal transplant recipients University of Health Sciences Bursa Yuksek Ihtisas Trainning and Reseach Hospital 
Complete blood count and biochemical test results of in the normal range. the participants are given in Table 2 and they were all

Table2: Blood test results of the participants

\begin{tabular}{|c|c|c|}
\hline & Mean & St. Deviation \\
\hline Hemoglobin & 12.9620 & 1.95123 \\
\hline Hematocrit & 39.1040 & 5.61270 \\
\hline WBC & 6.9492 & 2.38611 \\
\hline PLT & 216.634 & 66.0871 \\
\hline $\mathrm{MCV}$ & 85.5360 & 6.1516 \\
\hline Glucose & 101.500 & 38.8992 \\
\hline BUN & 21.9844 & 9.5554 \\
\hline Creatinine & 1.2020 & .42340 \\
\hline GFR & 71.5956 & 24.6235 \\
\hline Sodium & 138.20 & 3.6213 \\
\hline Potassium & 4.3500 & .48174 \\
\hline Albumin & 45.6580 & 4.7859 \\
\hline
\end{tabular}

\section{Discussion}

In this study we interrogated the symptoms reported in COVID-19 cases in previous publications (7-12). Two of the most frequent symptoms of COVID-19, fever and shortness of breath, were not detected in any of our patients. We collected nasopharyngeal swabs from all of our renal transplant patients who were in the risk group due to the immunosuppressive treatment they had undergone and we performed PCR tests. COVID-19 tests were negative in all our patients. 
In a study from Italy, it was reported that two renal transplant recipients were hospitalized due to COVID19 pneumonia (13). According to this article, when a 75 year-old male patient and 52 year-old female patient were admitted to the hospital with complaints of coughing, shortness of breath, myalgia and high fever, graft functions of the male patient were assessed to be normal but acute renal damage was detected in the female patient. These patients whose immunosuppressive treatments were abandoned as extensive icy glass appearance presented in their lungs in the thorax CT and their nasopharyngeal swabs were positive, died of COVID-19 as their situation deteriorated with time.

In another article, it was reported that there was a history of hypertension in $34(94 \%)$ of 36 adult patients with COVID-19 positivity, $75 \%$ of whom had undergone renal transplantation from cadavers, with diabetes mellitus in 25 (69\%) and smoking history in 13 of the patients (36\%). The most frequent symptoms were fever in 21 patients (58\%) and diarrhea in 8 patients (22\%). While 8 patients (22\%) in stable condition who did not have major respiratory symptoms were followed-up at home, 28 patients (78\%) were followed-up in the hospital. $10(28 \%)$ of 36 renal recipients died within an average of 21 days of follow-up. Two of these patients had been sent home at the beginning (14). In an article from England, 7 renal recipients who were COVID-19 positive were reported. Two of them underwent ambulatory treatment and five of them were treated in the intensive care unit. All of these patients presented respiratory symptoms and high fever, and an increase was detected in lymphopenia, C-reactive protein (CRP), D-dimer, ferritin and troponin levels in severe cases. 1 of these 7 patients died and it was recommended that renal transplantation must not be performed during the COVID-19 pandemic, especially in elderly patients with comorbidities (15).
COVID-19 cases who underwent renal transplantation present a quite mortal course with comorbidities and the effect of the immunosuppressive treatment. For this reason, serious precautions must be taken in this group of patients. There were comorbid diseases in most of our renal transplant patients as well, such as hypertension and diabetes mellitus, and in none of them was COVID-19 positivity detected. The reason for this may be that the virus reached Turkey relatively later than other countries and we had the chance to take the necessary precautions earlier by observing other examples throughout the world (16-22). In Turkey, early closure of schools, lockdown for the younger population up to 20 years old, who are likely to increase the infection risk, and for the population older than 65 years, which is the most critical risk group, significantly hindered the spread of the virus and the increase in numbers of deaths compared to European countries with a similar population. In parallel with this, we warned our renal transplant patients not to go out unless necessary and we provided for all their controls and follow-ups at home by our home health care services. We reached our transplant patients periodically and performed their examinations and treatments at home. All COVID-19 symptoms were interrogated and necessary examinations were performed. It can be asserted that this service contributed to preventing viral infection of the patients.

In conclusion, there is limited information on the risk and results of COVID-19 infection in renal transplant recipients who may be at high risk due to long-term immunosuppression, comorbidities and residual chronic renal failure. In this study, renal transplant patients were followed-up at home by home care services and thus kept away from the hospital environment. We assume that Covid-19 negativity during the first wave in our patients was a success of home care services. 


\section{Conflict of interest}

The authors declare that there is no conflict of interest.

\section{References}

1- World Health Organization (WHO), Geneva, Switzerland. WHO Coronavirus Disease (COVID-19) Dashboard. Available at: https://covid19.who.int/ [Accessed Jun 29, 2020].

\section{2-T.C.Sağlık Bakanlığı, https://covid19.saglik.gov.tr/}

3-Ma, Y., Diao, B., Lv, X., Zhu, J., Liang, W., Liu, L., ... \& Ding, G. (2020). 2019 novel coronavirus disease in hemodialysis (HD) patients: Report from one HD center in Wuhan, China. MedRxiv.

4- Naicker, S., Yang, C. W., Hwang, S. J., Liu, B. C., Chen, J. H., \& Jha, V. (2020). The Novel Coronavirus 2019 epidemic and kidneys. Kidney International, 97(5), 824-828.

5-Alberici, F., Delbarba, E., Manenti, C., Econimo, L., Valerio, F., Pola, A., ... \& Venturini, M. (2020). A single center observational study of the clinical characteristics and short-term outcome of 20 kidney transplant patients admitted for SARS-CoV2 pneumonia. Kidney international.

6-Zhou, F., Yu, T., Du, R., Fan, G., Liu, Y., Liu, Z., ... \& Guan, L. (2020). Clinical course and risk factors for mortality of adult inpatients with COVID-19 in Wuhan, China: a retrospective cohort study. The lancet.

7-Menni, C., Valdes, A. M., Freidin, M. B., Sudre, C. H., Nguyen, L. H., Drew, D. A., ... \& Visconti, A. (2020). Real-time tracking of self-reported symptoms to predict potential COVID-19. Nature medicine, 1-4.

8-Cascella, M., Rajnik, M., Cuomo, A., Dulebohn, S. C., \& Di Napoli, R. (2020). Features, evaluation and treatment coronavirus (COVID-19). In Statpearls [internet]. StatPearls Publishing.

9-Zhai, P., Ding, Y., Wu, X., Long, J., Zhong, Y., \& Li, Y. (2020). The epidemiology, diagnosis and treatment of COVID-19. International journal of antimicrobial agents, 105955.

10-Wan, S., Xiang, Y., Fang, W., Zheng, Y., Li, B., Hu, Y., ... \& Huang, X. (2020). Clinical features and treatment of COVID-19 patients in northeast Chongqing. Journal of medical virology.

11-Gautier, J. F., \& Ravussin, Y. (2020). A New Symptom of COVID-19: Loss of Taste and Smell. Obesity, 28(5), 848-848.
12-Jin, X., Lian, J. S., Hu, J. H., Gao, J., Zheng, L., Zhang, Y. M., ... \& Yu, G. D. (2020). Epidemiological, clinical and virological characteristics of 74 cases of coronavirus-infected disease 2019 (COVID-19) with gastrointestinal symptoms. Gut, 69(6), 1002-1009.

13-Gandolfini, I., Delsante, M., Fiaccadori, E., Zaza, G., Manenti, L., Degli Antoni, A., ... \& Maggiore, U. (2020). COVID-19 in kidney transplant recipients. American Journal of Transplantation.

14-Akalin, E., Azzi, Y., Bartash, R., Seethamraju, H., Parides, M., Hemmige, V., ... \& Liriano-Ward, L. (2020). Covid-19 and kidney transplantation. New England Journal of Medicine.

15- Banerjee, D., Popoola, J., Shah, S., Ster, I. C., Quan, V., \& Phanish, M. (2020). COVID-19 infection in kidney transplant recipients. Kidney International. 16-Rothan, H. A.,\&Byrareddy, S. N. (2020). Theepidemiologyandpathogenesis of coronavirusdisease (COVID-19) outbreak. Journal of autoimmunity, 102433.

17- Bogoch, I. I.,Watts, A., Thomas-Bachli, A., Huber, C., Kraemer, M. U., \&Khan, K. (2020). Pneumonia of UnknownEtiology in Wuhan, China: Potential for International Spread Via Commercial Air Travel. Journal of Travel Medicine.

18-Lu, H.,Stratton, C. W., \&Tang, Y. W. Outbreak of Pneumonia of Unknown Etiology in Wuhan China: theMysteryandtheMiracle. Journal of MedicalVirology.

19- Zhao, S., Lin, Q., Ran, J., Musa, S. S., Yang, G., Wang, W., ... \&Wang, M. H. (2020). Thebasicreproductionnumber of novelcoronavirus (2019-nCoV) estimation based on exponential growth in the early outbreak in China from 2019 to 2020: A replytoDhungana. International Journal of InfectiousDiseases, 94, 148-150.

20-Du Toit, A. (2020). Outbreak of a novelcoronavirus. Nature ReviewsMicrobiology, 18(3), 123-123.

21-Ren, L. L.,Wang, Y. M., Wu, Z. Q., Xiang, Z. C., Guo, L., Xu, T., ... \&Li, H. (2020). Identification of a novelcoronaviruscausing severe pneumonia in human: a descriptivestudy. Chinesemedicaljournal.

22-Lu, H. (2020). Drug treatment options for the 2019- new coronavirus (2019nCoV). Biosciencetrends, 14(1), 69-71. 